of increasing public awareness and education on earlier presentation of suspicious symptoms.

\section{P156 NAEDI LUNG CANCER AWARENESS CAMPAIGN IN LONDON}

doi:10.1136/thoraxjnl-2011-201054c.156

${ }^{1} \mathrm{~A}$ Safa, ${ }^{2} \mathrm{~A}$ Berry, ${ }^{2} \mathrm{H}$ Ali, ${ }^{3} \mathrm{~J}$ Car, ${ }^{2} \mathrm{E}$ F Bowen. ${ }^{1}$ Brook Green Medical Centre Hammersmith \& Fulhma PCT, London, UK; ${ }^{2}$ Charing Cross Hospital, Imperial College Healthcare NHS Trust, London, UK; ${ }^{3}$ Hammersmith \& Fulham PCT, London, UK

Background Lung cancer is the leading cause of cancer mortality in the UK. Survival rates over the last 30 years have not significantly improved. The NAEDI initiative is working to promote early diagnosis to improve stage at presentation and increase survival rates. Objectives The project in Hammersmith \& Fulham focused on the three most deprived wards, population 32 000). The aims were to increase public awareness and intention to act on symptoms by $10 \%$, GP chest x-ray referrals by $15 \%$, and Two Week Rule (TWR) referrals and, ultimately, earlier stage at diagnosis by $10 \%$.

Methods Nine general practices in the target area received educational seminars on NICE guidelines, project details and aims. 40 pharmacies, 25 smoking cessation advisors and 30 district nurses received education on symptoms, communication and management skills, and were provided with campaign materials. The public campaign involved training 42 health champions in key messages regarding alarm symptoms. Face-to-face contact was made with 1300 people. Local advertising had an estimated reach of three million people. The campaign ran from February to July 2011.

Results Data on public perceptions were collected with a validated form of the Cancer Awareness Measure. On average there was a 12\% increase in unprompted recall of the commonest lung cancer symptoms, and a $26 \%$ increase in intention to make urgent GP contact for such symptoms. Data from the 9 practices during the campaign period in 2011 compared to the same period in 2010 showed a rise in chest x-ray referrals from 350 to 463 (32\% increase). In 2010 there were no TWR referrals and seven lung cancer diagnoses. For 2011, there was 1 TWR referral (lung cancer) and 10 new lung cancers diagnosed.

Conclusions Community engagement has been a success locally with active involvement of trained champions into future public health work streams. Data collection again next year is required to evaluate whether the increase in chest $\mathrm{x}$-ray referral rates is maintained and translated into earlier stage at diagnoses. The success of primary care engagement needs roll out across the borough and embedding into future service provision to ensure sustainability.

\section{P157 FOLLOW-UP OF PULMONARY NODULES: FOLLOWING THE FLEISCHNER RADIOLOGY GUIDELINES}

doi:10.1136/thoraxjn-2011-201054c.157

S Selvaraj, L Parry, K Garbett, J McAdam, T R Naicker, K S Srinivasan, H Moudgil. Princess Royal Hospital, Telford, UK

Background $0.1 \%$ of chest films and $1 \%$ of chest CT show incidental pulmonary nodule(s). While some of these are acted on immediately, others are followed up where the nodules are small and there are no risk factors (never smokers, age $<35$ years, other malignancies). Fleischner Society recommends interval CT follow-up of pulmonary nodules [MacMahon $\mathrm{H}$ et al, Radiology 2005], but whether we adhere to these is uncertain and presently we report our practices based on this.

Methods Over a 4-year period to July 2009, 145 of all cases discussed at our lung multidisciplinary (MDT) meeting had a pulmonary nodule(s). They are either considered for immediate action or subsequent follow-up. Mean (SD, range) year age for this cohort was $65(11.04,37$ to 86$)$ of which 82 were male and 63 female. Nodules were classified as single or multiple, and by initial size of the largest nodule where multiple.

Results Of the 145 cases, 97 had single nodule and 48multiple. Of these, respectively ( $\mathrm{n}=$ absolute number) these were $=4 \mathrm{~mm}$ (13), $>4-6 \mathrm{~mm}(20),>6-8 \mathrm{~mm}(22),>8 \mathrm{~mm}$ (87) with do data in three. There were no follow-up data for 14, of whom 7 had refused subsequent follow-up, thad been discussed at MDT but no more proposed, and three died. Where there were data for follow up, 69 $(53 \%)$ followed Fleischner's guidelines on case selection and recommended interval scanning and 62 (47\%) did not usually but not exclusively due to delayed imaging intervals. Classification by initial nodule size and whether appropriate follow-up or not and sub-divided by whether malignant or not is shown in the enclosed Abstract P157 table 1 ( $n=119$, six still on active follow-up). Figures include those managed with PET scans whether under taken immediately (11) or later during follow-up (34). Of the entire cohort 28 had malignant disease of which 16 underwent surgery and 12 had an input through oncology. Of these, five had been identified as malignant because of increased size (2) or number (2) or because the nodule was persistent (1) but none had change in attenuation. Other cases where concerns were raised were benign $(n=10)$ but were similarly being considered due to multiplicity of nodules (3), uncertainty (1) and a persistent opacity (1) but exact reasoning was not available in the five others.

\section{Abstract P157 Table 1}

\begin{tabular}{lllllll}
\hline Appropriate timing & Outcome & $\mathbf{5 4} \mathbf{~ m m}$ & $\mathbf{> 4 - 6} \mathbf{~ m m}$ & $\mathbf{> 6 - 8} \mathbf{~ m m}$ & $\mathbf{> 8} \mathbf{~ m m}$ & Totals \\
\hline Yes & Benign & 4 & 4 & 4 & 30 & 42 \\
& Malignant & 1 & 3 & 1 & 16 & 21 \\
\multirow{2}{*}{ No } & Benign & 6 & 10 & 14 & 19 & 49 \\
& Malignant & - & - & - & 7 & 7 \\
\hline
\end{tabular}

Conclusion Data show that we conform to the Fleischner guidelines in approximately half the cases and these identified 15 who required further specific investigation and of which five had malignant disease. Although this is a small number of those followed up it supports the practice of follow-up but which still needs to better protocol driven.

\section{P158 END OF LIFE CARE FOR LUNG CANCER; CAN WE IDENTIFY THOSE IN NEED OF SUPPORT}

doi:10.1136/thoraxjnl-2011-201054c.158

S M Jones, J Gallagher, T Sweeney, E Pugh, A Mclver, V Kelly, M J Walshaw M Ledson. Liverpool Heart and Chest Hospital, Liverpool, UK

Introduction and Objectives The End of Life Care Strategy advocates the identification and support of patients approaching the end of their lives, and to aid this, social care benefits can be awarded to those with a life expectancy $<6$ months by completion of a DS1500 form. Many lung cancer patients fall into this category: we wished to look at the use of this enhanced support mechanism in our busy lung cancer unit.

Method We looked at the use of DS1500 in the initial period for 100 consecutive outpatients with lung cancer diagnosed through our rapid access clinic in 2007 (mean age 73 years [SD 8], mean PS=2 [IOR 2], 47 females), comparing its use with survival and histological tumour type. Mortality data were obtained from the national registry.

Results Twenty two had a DS1500 completed (median 26 days [20] from presentation): there was no difference in age, sex or PS between these and the remainder. Of the 75 with a tissue diagnosis, 
only those with non-small cell cancer were more likely to receive a form $\left(16 / 22\right.$ vs $\left.30 / 78 \chi^{2}=8.1, p=0.004\right)$. Only three patients with a clinical diagnosis and three with small cell received forms (both $\mathrm{p}=\mathrm{NS}$ ). Overall median survival was 216 days [IOR 405], with 57 alive at 6 months and 33 at 1-year. Median survival was 193 [268] days for those with DS1500 compared with 224 [458] in the remainder $(p=N S)$, and $31(57 \%)$ of those who died within 6 months did not benefit from early DS1500 status.

Conclusion While the DS1500 use was appropriate, the number identified represented less than a half of those who died within 6 months of presentation, and those with the poorest prognosis (small cell type or a clinical diagnosis) did not benefit the most. It seems we are missing an opportunity to support this unfortunate group of patients at the time of their greatest need.

\section{P159 RATE OF CHEST X-RAYS (CXR) TWELVE MONTHS PRIOR DIAGNOSIS OF LUNG CANCER}

doi:10.1136/thoraxjnl-2011-201054c.159

A Dwarakanath, M E J Callister. Leeds Teaching Hospitals NHS Trust, Leeds, UK

Introduction UK 5 year survival from lung cancer lags behind other developed nations, and one suggested reason is late stage at diagnosis. Efforts are therefore underway to encourage patients to present earlier to primary care with chest symptoms, and to encourage earlier referral in primary care for CXR or clinic review. Stage at presentation varies between geographical areas, suggesting variability in referral even within UK regions. The reasons underlying this variability are unknown, but one possible explanation could be different patient behaviours or clinical practice in primary care.

Aim To analyse the number of CXRs done 12 months prior to a confirmed diagnosis of lung cancer according to stage at presentation. Method We reviewed all patients with lung cancer discussed at the Lung MDT between December 2008 and May 2009. Cases of Small Cell Lung Cancer were excluded. The stage (IASLC 6th system), and number of CXRs performed within 12 month prior to diagnosis were recorded. Numbers of CXRs for each group were compared using Kruskal-Wallis test with Dunn's post-test comparison.

Results 223 NSCLC (stage $1 \& 2=61$, stage $3=61$, stage $4=101$ ) and 32 SCLC (limited-12 \& extensive-20) were diagnosed. Median, IOR and range of number CXRs in the previous 12 months are displayed in Abstract P159 table 1. Patients presenting with stage IV lung cancer had undergone significantly fewer CXRs in the year prior to diagnosis compared to patients diagnosed with stage I/II lung cancer $(p<0.05)$ and those diagnosed with stage III disease $(p<0.05)$. There was no significant difference between the stage I/II and stage III disease.

Abstract P159 Table 1

\begin{tabular}{llll}
\hline & Median & IQR & Range \\
\hline I\&II $(\mathrm{n}=61)$ & 2 & $0-3$ & $0-14$ \\
III $(\mathrm{n}=61)$ & 2 & $1-3$ & $0-12$ \\
IV $(\mathrm{n}=101)$ & 1 & $0-2$ & $0-7$ \\
\hline
\end{tabular}

Conclusion Patients with late stage disease (Stage IV) appear to have fewer CXRs in the year prior to diagnosis than patients presenting with earlier disease. This may represent a reluctance to seek medical review for persistent respiratory symptoms, or reluctance among GPs to refer for CXR. Ongoing analysis is investigating rates of GP consultation and antibiotic prescription among these patients to try to discriminate between then possible explanations.

\section{P160 TURNING ROUND LUNG CANCER CARE IN LIVERPOOL: A 15-YEAR AUDIT}

doi:10.1136/thoraxjnl-2011-201054c.160

A Nazir, T A Aljemmali, S M Kazmi, D Nazareth, K Mohan, C Smyth, M Ledson, M Walshaw. Liverpool Hear and Chest Hospital NHS Foundation Trust, Liverpool, UK

Background Liverpool has the highest incidence of lung cancer in England and Wales, and a citywide audit in 1996 identified significant deficiencies in the secondary care sector management of suspected cases. As a result of this, major reforms were made to benefit the majority of Liverpool residents in 1999, including the collaboration of competing hospitals to provide a unified service, a dedicated one stop rapid access clinic with same-day CT and bronchoscopy facilities, the employment of lung cancer specialist nurses, and parallel clinics for speedy onward referral. We have continued to refine this service, the largest in the region, and wished to compare outcomes to date.

Methods We compared the results of the service at three time points: at the 1996 audit, immediately after the inception of the service in 2001 and in 2010.

Results The results are summarised in the Abstract P160 table 1. In addition by 2010, two thirds had CT and bronchoscopy (6 EBUS) on the same day as their rapid access clinic appointment, and 48 underwent EBUS (mean wait 5 days). The average waiting time for PET scan in 2010 was 10 days.

\section{Abstract P160 Table 1}

\begin{tabular}{llll}
\hline Year & $\mathbf{1 9 9 6}$ & $\mathbf{2 0 0 1}$ & $\mathbf{2 0 1 0}$ \\
\hline Total no & 123 & 134 & 408 \\
Mean time to see chest physician (days) & 14.8 & 4.4 & 4.0 \\
Number presenting via A\&E (\%) & 67 & 42.5 & 38 \\
Proportion undergoing Bronchoscopy (\%) & 54 & 85.8 & 88.7 \\
$\begin{array}{l}\text { Average time interval to bronchoscopy } \\
\text { (days) }\end{array}$ & 14.6 & 4.4 & 1.23 \\
$\begin{array}{l}\text { No of patients undergoing staging CT scan } \\
\text { (\%) }\end{array}$ & $60(\mathrm{~N}=74)$ & $83.5(\mathrm{~N}=112)$ & $99.2(\mathrm{~N}=405)$ \\
Histological diagnosis achieved (\%) & $61(\mathrm{~N}=75)$ & $75.3(\mathrm{~N}=104)$ & $80.85(\mathrm{~N}=330)$ \\
Surgical resection rate (\%) & $6.50(\mathrm{~N}=8)$ & $8.20(\mathrm{~N}=11)$ & $16.8(\mathrm{~N}=69)$ \\
\hline
\end{tabular}

Conclusion This study confirms that the care of lung cancer patients in Liverpool has improved since the introduction of these new services, which we encourage other units to emulate.

\section{P161 COMBINED 18F-FDG PET/CT AS PART OF A SURVEILLANCE PROGRAMME OF PATIENTS WITH NEWLY DIAGNOSED PRE- INVASIVE ENDOBRONCHIAL LESIONS DETECTS SYNCHRONOUS LUNG CANCERS}

doi:10.1136/thoraxjnl-2011-201054c.161

${ }^{1} \mathrm{~L} J$ E Smith, ${ }^{2} \mathrm{~K}$ Kayani, ${ }^{2} \mathrm{~J}$ Bomanji, ${ }^{2} \mathrm{~A}$ M Groves, ${ }^{3} \mathrm{~B}$ Carroll, ${ }^{3} \mathrm{~N}$ Navani, ${ }^{3} \mathrm{~J}$ P George, ${ }^{3} \mathrm{~S}$ M Janes. ${ }^{1}$ University College Hospital, London, UK: ${ }^{2}$ Institute of Nuclear Imaging, UCL, London, UK; ${ }^{3}$ Centre for Respiratory Research, UCL, London, UK

Background Patients with bronchial pre-invasive lesions represent a significant management challenge due to the risk of lung cancer both at the site of known dysplasia and at remote sites within their lungs. The role of combined positron emission tomography/CT (PET/CT) in those patients is unknown.

Aims To evaluate the diagnostic and clinical utility of 18F-FDG PET/CT in a surveillance programme for patients with pre-invasive endobronchial lesions. This was defined as the ability of abnormal 18F-FDG uptake to detect invasive bronchial carcinomas adjacent to known pre-invasive lesions or at remote sites in the lung. The 